\title{
Investigación y desarrollo en catálisis en el Instituto Nacional de Investigaciones Nucleares*
}

\author{
Luis Escobar Alarcón, ** Suilma Marisela Fernández Valverde, ${ }^{* * *}$ \\ Jaime Jiménez Becerril,,** Régulo López Callejas, ${ }^{* *}$ \\ Raúl Valencia Alvarado, ${ }^{* *}$ Raúl Pérez Hernández****
}

RESUMEN: Se describen las actividades de investigación en el área de catálisis que se realizan en el Instituto Nacional de Investigaciones Nucleares (ININ) y se refieren las atribuciones que por mandato legal tiene el Instituto. Se incluyen las líneas de investigación, contribuciones y los principales logros en este campo obtenidos a través de los años. También se presenta información sobre la infraestructura con que cuenta el Instituto para llevar a cabo investigación sobre catálisis. Se menciona la importancia del trabajo de colaboración nacional e internacional, así como la formación de recursos humanos. Por último, se presentan las perspectivas de investigación y desarrollo en nuestra institución en el campo de la catálisis.

PALABRAS CLAVE: Catálisis, nanocatálisis, electrocatálisis, fotocatálisis, radiocatálisis, nanomateriales, energía, ambiente.

ABSTRACT: This manuscript shows the activities in the catalysis field performed in the National Institute of Nuclear Research (Spanish acronym: ININ), and briefly are included the goals and attributions that by legal mandate has the Institute. It includes research lines, contributions, and main achievements in this field obtained through the years. In addition, information about the infrastructure available in the Institute to perform the research on catalysis is presented. The importance of the national and international collaborations, as well as, the projects in this area, and human resources training are emphasized. Finally, we present the research and development prospects on catalysis in our Institution in the catalysis field.

KEYWORDS: Catalysis, nanocatalysis, electrocatalysis, photocatalysis, radiocatalysis, nanomaterials, energy, environment.

\section{Introducción}

El Instituto Nacional de Investigaciones Nucleares (ININ) es un organismo descentralizado del gobierno federal, dependiente de la Secretaría de Energía (SENER). Fue creado mediante la Ley Reglamentaria del Artículo 27 Constitucional en Materia Nuclear, que en el capítulo V, artículo 43, establece que

Recibido: 23 de enero de 2017. Aceptado: 31 de marzo de 2017.

* Agradecemos a Mundo Nano. Revista Interdisciplinaria en Nanociencias y Nanotecnología el permitirnos difundir las actividades en catálisis que desarrolla el ININ.

** Departamento de Física. Instituto Nacional de Investigaciones Nucleares (ININ), carretera México-Toluca S/N, La Marquesa, C.P. 52750, Ocoyoacac, Edo. de México, México.

*** Departamento de Química, ININ.

**** Departamento de Tecnología de Materiales, ININ.

Correspondencia: (raul.perez@inin.gob.mx). 

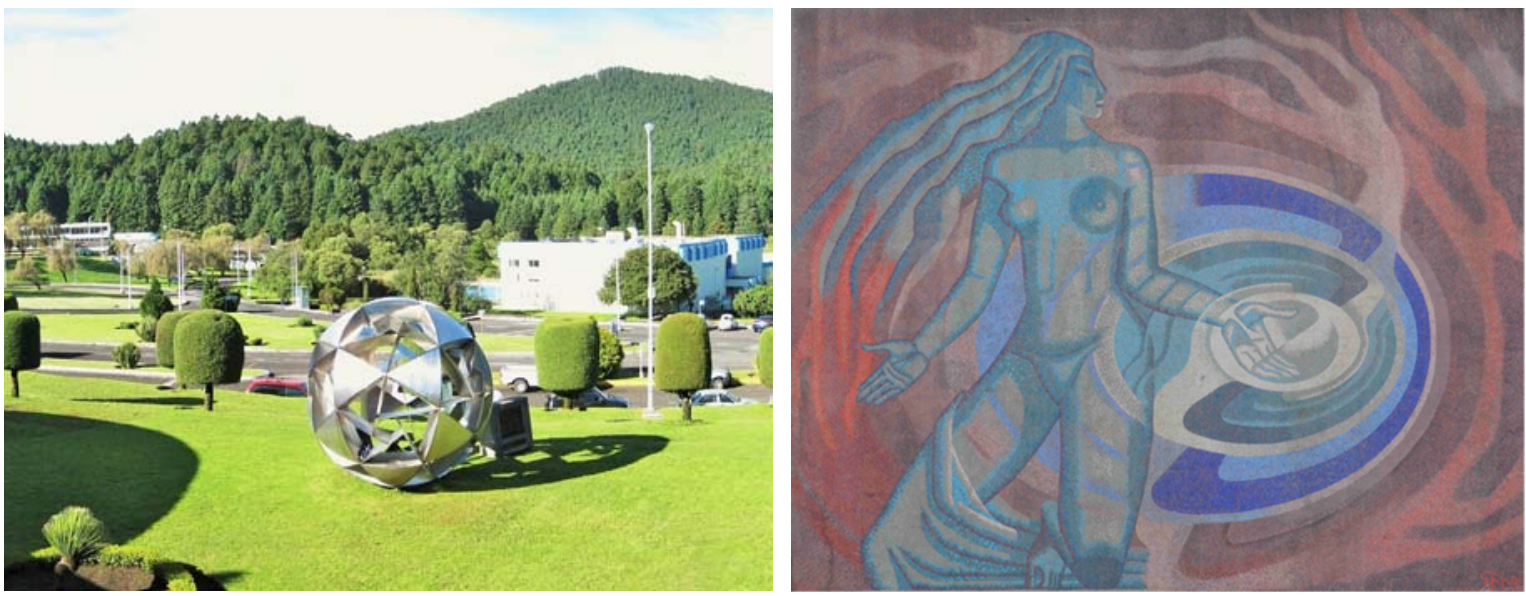

FIGURA 1. Vista panorámica (izq.) y mural (dcha.): "La creación de la energía”, de Francisco Eppens, en el edificio del reactor nuclear TRIGA del Centro Nuclear.

para el cumplimiento de su objeto, el ININ tendrá, entre otras atribuciones, las siguientes: realizar e impulsar las actividades que conduzcan al desarrollo científico y tecnológico en el campo de las ciencias y tecnologías nucleares, así como promover la transferencia, adaptación y asimilación en esta materia; prestar asistencia técnica a las dependencias y entidades públicas y privadas que lo requieran, en el diseño, construcción y operación de instalaciones radiactivas; realizar actividades de investigación y desarrollo relativas a las aplicaciones y aprovechamiento de sistemas nucleares y materiales radiactivos para usos no energéticos requeridos para el desarrollo nacional; además, promoverá las aplicaciones de las radiaciones y los radioisótopos en sus diversos campos.

Las líneas de investigación que el ININ tiene para cumplir tal propósito, aprobadas por su Órgano de Gobierno, son: aplicaciones de las radiaciones a los sectores industrial, salud y agropecuario, aplicaciones de los aceleradores de partículas, ciencias nucleares, ecología y protección del medio ambiente, fuentes energéticas, gestión de desechos radiactivos, materiales nucleares y radiactivos, química y radioquímica, radiobiología y genética, seguridad nuclear y radiológica, tecnología de reactores nucleares. En el marco de estas líneas de investigación y buscando impulsar la incursión en temas emergentes, con el propósito de aprovechar las oportunidades coyunturales que representan y que abren nuevos espacios para el progreso y bienestar social, en línea con el Programa Especial de Ciencia, Tecnología e Innovación (PECITI) 2014-2018, que se desprende del objetivo 3.5 del Plan Nacional de Desarrollo 2013-2018, que a la letra dice: "Hacer del desarrollo científico, tecnológico y la innovación pilares para el progreso económico y social sostenible", el propósito de los objetivos rectores del PECITI es guiar en la 
transición del país hacia una economía basada en el conocimiento. En particular, los temas prioritarios y de competencia para el ININ serían:

- Ambiente: gestión integral del agua, seguridad hídrica y derecho al agua.

- Conocimiento del Universo: estudios de física, matemáticas, química y sus aplicaciones.

- Desarrollo tecnológico: desarrollo de materiales avanzados y desarrollo de nanomateriales y de nanotecnología

- Energía: desarrollo y aprovechamiento de energías renovables y limpias.

En el ININ se realizan proyectos de investigación con el objetivo de desarrollar nuevos nanomateriales con aplicaciones catalíticas para la generación de combustibles alternos al petróleo y para la eliminación de contaminantes orgánicos recalcitrantes presentes en el agua mediante procesos de oxidación avanzada. En el primer caso, se desarrollan proyectos de investigación para atacar uno de los problemas ocasionados por nuestra dependencia del petróleo; una de las perspectivas y estrategias a seguir para la solución de estos problemas está encaminada hacia un sistema energético basado en el hidrógeno $\left(\mathrm{H}_{2}\right)$, el cual es considerado como el combustible del futuro o la "economía del hidrógeno". El $\mathrm{H}_{2}$ proveerá a la humanidad de un recurso energético eficaz y mucho más limpio, que asegure su continuo y futuro desarrollo. El proceso más aceptado para la obtención del $\mathrm{H}_{2}$ es el reformado del bioalcoholes (metanol, etanol) y metano proveniente del biogás, en presencia de catalizadores o electrocatalizadores apropiados, en estos casos el $\mathrm{CO}_{2}$ emitido está considerado dentro del ciclo del carbono. El hidrógeno generado puede ser utilizado como fuente de energía en las celdas de combustible (FC-fuel cell) donde los únicos subproductos son agua y calor. La otra vertiente de estudio en el ININ, asociada con el crecimiento poblacional, industrial y la demanda de servicios, está relacionada con la contaminación del agua con compuestos orgánicos, altamente tóxicos, químicamente estables y persistentes, con efectos adversos para la salud humana y el medio ambiente. En particular, en los últimos años ha crecido el interés en los denominados "contaminantes emergentes" que se encuentran presentes en aguas residuales, así como en aguas superficiales e incluso potable; estos contaminantes tienen su origen en diversas sustancias de uso cotidiano como son: fármacos, de cuidado personal e incluso hormonas. Es importante señalar que las plantas de tratamiento de aguas residuales no están diseñadas para remover sustancias complejas de este tipo. Por tal motivo, se hace necesaria la búsqueda de métodos eficaces para la eliminación de estos contaminantes presentes en medio acuoso. Una alternativa es el uso de los procesos de oxidación avanzada (POA), que parten de procesos fisicoquímicos capaces de producir cambios significativos en la estructura del contaminante logrando su degradación parcial o completa. Dentro 
de estos procesos se encuentra la fotocatálisis heterogénea, considerada como una tecnología promisoria, eficiente y limpia, que se define como "el cambio en la velocidad de una reacción química o su iniciación debido a la exposición a la radiación ultravioleta, visible o infrarroja en presencia de una sustancia, el fotocatalizador, que absorbe cuantos de luz y participa en la transformación química de los participantes de la reacción". La fotocatálisis se basa en el uso de radiación electromagnética que incide sobre un material sólido, generalmente un semiconductor, capaz de absorber radiación con energía igual o mayor a la de su energía de banda prohibida, generando especies oxidantes, que permiten la degradación parcial o completa de contaminantes hasta llevarlos a la mineralización total, transformándolos en $\mathrm{CO}_{2}$ y $\mathrm{H}_{2} \mathrm{O}$. Las reacciones catalizadas inducidas por radiaciones ionizantes o radiocatálisis, es un proceso que combina en sí mismo varias reacciones radiocatalíticas que no están entendidas del todo, por lo que se encuentran en la etapa de investigación.

A través del trabajo de investigación realizado en el ININ, se pretende contribuir con alternativas que permitan eventualmente la mitigación de los problemas de contaminación ambiental; por un lado, mediante el desarrollo de nanocatalizadores para la producción y uso del hidrógeno como fuente alterna de energía limpia y, por el otro, desarrollando nanomateriales para la degradación de contaminantes presentes en el agua mediante POA utilizando luz solar; de esta forma, se promueve la cultura de desarrollo sustentable en materia de energía y ambiente.

\section{Líneas de investigación, desarrollo e innovación relacionadas con la catálisis}

Debido a la creciente necesidad de contar con desarrollos y energías sustentables, varios países incluyendo México pretenden disminuir sus emisiones contaminantes en los próximos años mediante el uso de biocombustibles, como se menciona en la Estrategia Nacional de Energía 2013-2027 a cargo de la Secretaría de Energía. En este contexto, un paso natural es buscar soluciones con los avances más recientes en ciencia, en particular, en la nanotecnología, disciplina que ofrece la posibilidad de desarrollar nuevos nanomateriales con estructuras preformadas (en forma de nanopartículas, nanorrodillos, nanotubos y nanohojas); es importante señalar que este tipo de nanocatalizadores no están siendo utilizados para la generación de $\mathrm{H}_{2}$ que puede ser empleado para generar energía limpia y son poco utilizados en procesos de oxidación avanzada, lo que le confiere cierto grado de originalidad al trabajo de investigación realizado en esta dirección. En este contexto, en el ININ se desarrollan líneas de investigación en el campo de catálisis, que involucran la síntesis, caracterización y aplicaciones de nanomateriales, los denominados nanocatalizadores:

- Diseño de nanocatalizadores unidimensionales (nanorods) con morfologías y planos cristalográficos preferenciales para la generación de 
hidrógeno, mediante la reacción de reformado de alcoholes y $\mathrm{CH}_{4}$.

- Desarrollo de nanomateriales para aplicarlos en celdas de combustible para la producción de hidrógeno como fotoelectrocatalizadores y electrocatalizadores. Los primeros para electrólisis de agua utilizando la luz solar y los segundos como nanomateriales para electrodos de: celdas de combustible, celdas regenerativas unificadas y celdas de carbonatos fundidos.

- Procesos de oxidación avanzada inducida por radiaciones modificando óxidos de titanio comerciales con metales de transición y soportados en matrices mesoporosas (MCM-41 y carbón), para la degradación de compuestos orgánicos contaminantes recalcitrantes y persistentes en efluentes acuosos, tal como residuos de la industria y plantas de tratamiento.

- Películas de $\mathrm{N}-\mathrm{TiO}_{2}$ obtenidas en un reactor de plasma sobre los sustratos de vidrio o silicio y su estudio en la remoción de contaminantes tóxicos en aire y agua, así como la generación de hidrógeno por electrólisis de agua.

- Preparación y caracterización de películas delgadas nanoestructuradas con aplicaciones fotocatalíticas.

- Desarrollo de nanomateriales basados en $\mathrm{TiO}_{2}$ y $\mathrm{V}_{2} \mathrm{O}_{5}$ para la degradación de moléculas orgánicas.

- Modificación de $\mathrm{TiO}_{2}$ y $\mathrm{V}_{2} \mathrm{O}_{5}$ con metales (Ag, $\mathrm{Bi}$, Au y $\mathrm{Zn}$ ) para la degradación fotocatalítica de fármacos utilizando radiación solar.

\section{Síntesis de nanomateriales}

- Preparación de nanocatalizadores bimetálicos 1D utilizando el método hidrotermal e impregnación clásica.

- Síntesis de materiales por sol-gel.

- Para catálisis inducida por radiaciones se modificaron algunos óxidos de titanio comerciales con metales de transición y se han soportado en matrices mesoporosas (MCM-41 y carbón).

- Síntesis de películas de dióxido de titanio dopadas con nitrógeno $\left(\mathrm{N}-\mathrm{TiO}_{2}\right)$ en plasmas de RF por el proceso de sputtering sobre sustratos de silicio y vidrio en las fases de anatasa y anatasa/rutilo.

- Preparación de nanomateriales mediante configuraciones no convencionales de ablación láser.

- Preparación de nanomateriales mediante configuraciones hibridas de ablación láser combinada con evaporación térmica y pulverización catódica.

- Preparación de nanomateriales mediante ablación láser en medio líquido.

- Preparación de nanomateriales híbridos formados por nanopartículas soportadas o embebidas en películas delgadas o en otros materiales. 
- Síntesis de electrocatalizadores nanoparticulados de metales nobles: $\mathrm{Pt}, \mathrm{Ru}, \mathrm{Pt}-\mathrm{Ru}$ obtenidos por descarboxilación de carbonilos en medio orgánico.

- Síntesis de electrocatalizadores por sol gel de aleaciones metálicas u óxidos: $\mathrm{Pt}-\mathrm{Ti}, \mathrm{Pt}-\mathrm{Ir}, \mathrm{Pt}_{\mathrm{x}} \mathrm{Ir}_{\mathrm{y}} \mathrm{O}_{\mathrm{z}}$ para funcionar en medio ácido.

- Síntesis por aleado mecánico de $\mathrm{TiCoO}_{3}$ de tamaño nanométrico, para reacciones de reducción y desprendimiento de oxígeno en medio alcalino.

\section{Caracterización de nanomateriales}

- Evaluación de las propiedades catalíticas de los nanocatalizadores 1D en reacciones de reformado de alcoholes y metano $\left(\mathrm{CH}_{4}\right)$.

- Reacción de electrólisis del agua siguiendo el desprendimiento de oxígeno (RDO) y reacción de desprendimiento de hidrógeno (RDH).

- Degradación de compuestos orgánicos contaminantes recalcitrantes y persistentes en efluentes acuosos por radiocatálisis.

- Caracterización de propiedades vibracionales de nanomateriales por espectroscopía infrarroja y espectroscopía Raman.

- Caracterización de propiedades ópticas mediante espectroscopía ultravioleta-visible, espectroscopía de reflectancia difusa y fotoluminiscencia.

- Caracterización morfológica, estructural y análisis químico por microscopía electrónica de barrido (SEM) y microscopía electrónica de transmisión (TEM) y espectroscopía de dispersión de energía (EDS), respectivamente; así como microscopía de fuerza atómica (AFM).

- Estudio de la cristalinidad de polvos y películas delgadas mediante difracción de rayos X y espectroscopía Raman.

FIGURA 2. Síntesis de micro-cepillos a base de Ag-ZnO y ZnO en forma de rods.
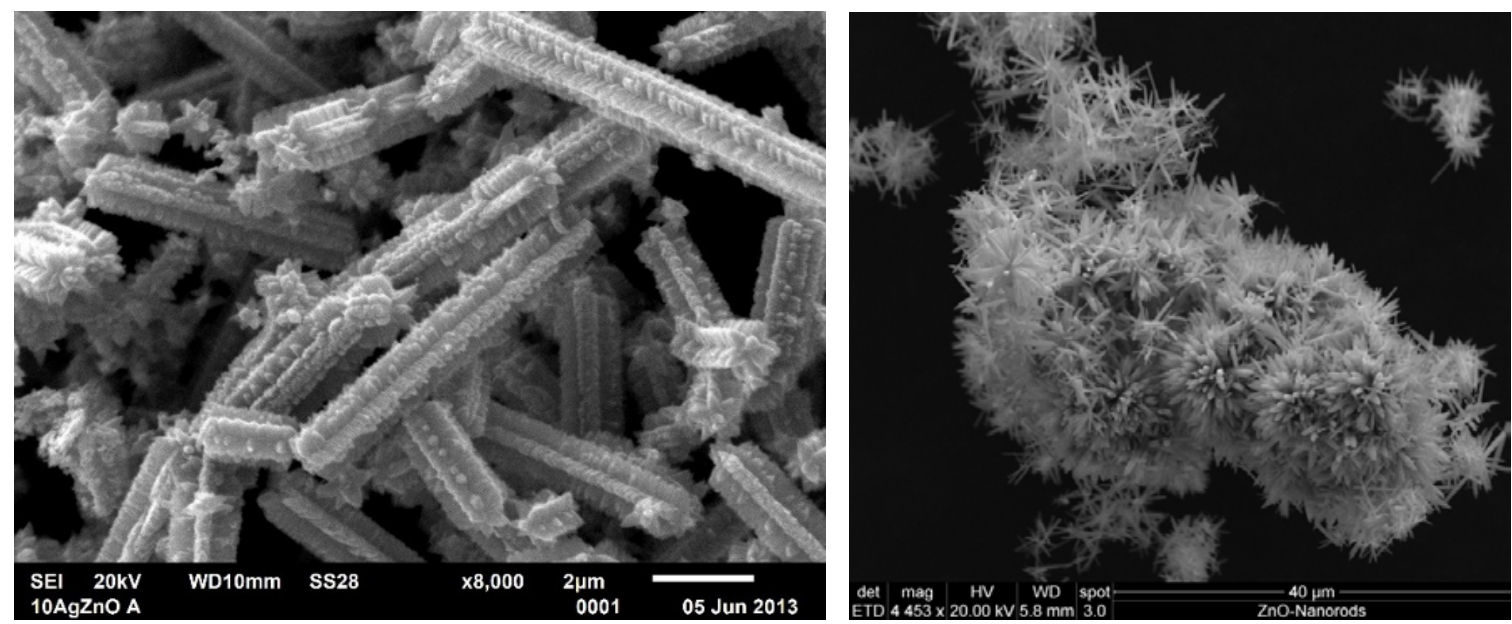
- Caracterización de nanomateriales mediante técnicas de haces de iones como espectroscopía de retrodispersión de Rutherford (RBS), análisis por dispersión elástica hacia adelante (EFA) y reacción nuclear (NR) entre otras.

- Caracterización del desempeño fotocatalítico en la degradación de moléculas orgánicas, como pigmentos y fármacos presentes en aguas residuales reales, utilizando radiación solar.

- Caracterización de las aleaciones de Pt obtenidas en medio orgánico las cuales mostraron un buen desempeño para la reacción de reducción de oxígeno en medio ácido.

- La evaluación electrocatalítica de los óxidos de molibdeno, cobalto y las mezclas de éstos mostraron un muy buen desempeño para la reacción de desprendimiento de oxígeno en medio alcalino.

- Las propiedades electrocatalíticas de los óxidos $\mathrm{TiCoO}_{3}$ y $\mathrm{Pt}_{\mathrm{x}} \mathrm{Ti}_{\mathrm{y}} \mathrm{Co}_{\mathrm{z}}$ presentan buena respuesta en reacciones de reducción y desprendimiento de oxígeno en medio alcalino.

\section{Aplicaciones}

- Aplicación de los catalizadores 1D desarrollados con tamaño y forma controlada en reacciones de reformado de alcoholes y biogás.

- Electrolizadores para celdas regenerativas unificadas evaluados en medio ácido y en medio básico, en colaboración con el Instituto de Investigaciones Eléctricas (IIE).

- Degradación de compuestos orgánicos contaminantes recalcitrantes y persistentes en efluentes acuosos, tales como residuos de la industria textil y plantas de tratamiento mediante fotocatálisis y radiocatálisis; así como en la generación de hidrógeno por separación de agua.

- Tratamiento de aguas residuales, en particular para la degradación de moléculas orgánicas, como pigmentos y fármacos presentes en aguas residuales reales, utilizando radiación solar.

- Aplicación de los electrocatalizadores de metales nobles en prototipos de celdas de combustible ácidas.

- Uso de los electrocatalizadores de metales de transición en celdas de combustible alcalinas.

- Aplicación de los óxidos de platino-iridio o de óxido de titanio dopado o de $\mathrm{TiCoO}_{3}$ en celdas regenerativas unificadas.

\section{Colaboraciones y proyectos}

Los grupos de investigación en catálisis en el ININ son multidisciplinarios y han adquirido experiencia en la preparación y manipulación de diferentes nanomateriales por métodos químicos, entre ellos, el método sol-gel para la 
síntesis de óxidos simples y compuestos, método hidrotermal para la obtención de óxidos unidireccionados. Se cuenta también con amplia experiencia en la preparación de películas delgadas y nanoestructuras utilizando técnicas basadas en plasmas. Adicionalmente, se tiene experiencia en la caracterización de catalizadores por técnicas físicas, químicas y nucleares. Lo anterior ha permitido establecer colaboraciones con investigadores de las siguientes instituciones:

\section{Nacionales:}

- Universidad Nacional Autónoma de México:

- Facultad de Química.

- Instituto de Investigaciones en Materiales.

- Centro de Ciencias Aplicadas y Desarrollo Tecnológico.

- Centro de Nanociencias y Nanotecnología.

- Instituto de Física.

- Facultad de Ingeniería.

- Centro de Física Aplicada y Tecnología Avanzada, Campus-Querétaro.

- Universidad Autónoma del Estado de México:

- Centro Conjunto de Investigación en Química Sustentable, dependiente de la Universidad Autónoma del Estado de México y la Universidad Nacional Autónoma de México.

- Facultad de Ciencias.

- Facultad de Química.

- Instituto Tecnológico de Toluca.

- Tecnológico de Tuxtla Gutiérrez, Chiapas.

- Universidad Tecnológica del Valle de Toluca.

- Universidad Autónoma Metropolitana Lerma.

- Universidad Autónoma Metropolitana Iztapalapa.

- Benemérita Universidad Autónoma de Puebla.

- Universidad Autónoma de Querétaro.

- Centro de Investigación y Desarrollo Tecnológico en Electroquímica, S.C. (CIDETEQ), Querétaro.

- Universidad Autónoma del Estado de Hidalgo: - Área Académica de Ciencias de la Tierra y Materiales.

- Centro de Investigación y Estudios Avanzados:

- Departamento de Química-Zacatenco.

- Grupo de sustentabilidad de los Recursos Naturales y Energía, Unidad Saltillo.

- Instituto de Investigaciones Eléctricas (actualmente INEEL-Instituto Nacional de Electricidad y Energías Limpias).

- Instituto Politécnico Nacional:

- Escuela Superior de Ingeniería Química e Industrias Extractivas. 


\section{Internacionales:}

- Universidad de Texas, Campus San Antonio.

- Laboratorios de Hidrógeno y de Materiales para altas Temperaturas. Depto. de Tecnología en Calor Nuclear del Instituto de Energía Atómica de Japón.

- Escuela Nacional Superior de Química de París (École Nationale Supérieure de Chimie de Paris ENSCP-Paris-Tech).

- Universidad de Columbia Británica, Vancouver, Canadá.

\section{Proyectos financiados por conACyT:}

- Síntesis y caracterización de electrocatalizadores bifuncionales para la reducción y desprendimiento de oxígeno y su desempeño en una celda regenerativa unificada (CB-2007-01).

- Desarrollo de catalizadores a base de $\mathrm{Cu}$ y $\mathrm{Ni}$ soportado en $\mathrm{ZrO}_{2}{ }^{-}$ $\mathrm{CeO}_{2}$ para la generación de hidrógeno mediante la reacción de reformado de metanol con vapor de agua en atmósfera oxidante ( $\mathrm{J}$ 023959).

- Efecto de la radiación gamma sobre la actividad fotocatalítica de óxidos de zinc y titanio (CB-83943).

- Oxidación de titanio por plasmas de radiofrecuencia inductivamente acoplados (CB-102466).

- Preparación de nanomateriales basados en $\mathrm{TiO}_{2}$ y $\mathrm{V}_{2} \mathrm{O}_{5}$ utilizando configuraciones no convencionales de ablación láser (CB-240998).

\section{Proyectos financiados por el ININ:}

- Estudio de factibilidad del uso de nanocatalizadores bimetálicos para la producción de hidrógeno, mediante el reformado de metanol (CA-409).

- Diseño de catalizadores unidimensionales para la generación de $\mathrm{H}_{2}$ mediante el reformado de metanol (CA-009).

- Desarrollo de catalizadores para su uso en la reducción catalítica selectiva de contaminantes atmosféricos y generadores de nuevos combustibles (CM-520).

- Estudio de las propiedades catalíticas para la eliminación de emisiones contaminantes, de metales soportados en óxidos mixtos (SC019).

- Preparación y caracterización de películas delgadas nanoestructuradas con aplicaciones fotocatalíticas (CB-402).

- Estudio de materiales con propiedades fotocatalíticas con posible aplicación en la degradación de resinas de intercambio iónico (CB407).

- Estudio de la degradación de residuos industriales de ensayos no destructivos por radiocatálisis (CB-504).

- Hidrógeno y materiales funcionales para celdas de combustible y re- 
generativas (CB-906).

- Prueba de materiales para la producción, almacenamiento y uso del hidrógeno (QU-001).

\section{Infraestructura}

El ININ cuenta con equipos para la síntesis, caracterización de propiedades físicas y evaluación de propiedades catalíticas que se listan a continuación:

\section{Síntesis}

- Dos fuentes de radiofrecuencia de $13.56 \mathrm{MHz}$, una con potencia de hasta $1200 \mathrm{~W}$ y otra con potencia hasta $600 \mathrm{~W}$, ambas con redes de acoplamiento automáticas, sistemas de vacío con base en bombas mecánicas y turbomoleculares, espectrómetros de masa para medición de presiones parciales hasta de 200 unidades de masa atómica, monocromadores/espectrógrafos, uno con una cámara de detección intensificada y el otro con detectores del tipo fotomultiplicadores.

- Sistema de depósito de películas delgadas por ablación láser, con dos láseres de Nd:YAG de $850 \mathrm{~mJ}$, con emisión en la línea fundamental y los dos primeros armónicos.

- Sistema de depósito de películas delgadas por pulverización catódica.

- Sistemas de depósito híbridos de ablación láser con evaporación térmica y pulverización catódica.

- Sistema de preparación de nanomateriales por ablación láser en medio líquido.

- Cajas de guantes para la obtención de nanocatalizadores en medio orgánico

- Equipo de microondas.

- Molinos de aleado mecánico.

\section{Caracterización de propiedades físicas y químicas}

- Sistema de caracterización de catalizadores BELCAT-B equipado con espectrómetro de masas BELMass y CATCryo.

- Equipo multitareas RIG-100.

- Cromatógrafos de gases.

- Espectroscopía infrarroja con transformada de Fourier en su modalidad de reflectancia difusa (DRIFT) y cámara catalítica.

- Equipos de microscopía electrónica para la caracterización de los materiales catalíticos.

- Potenciostato/galvanostato Princeton Applied Research, programador universal EG\&G Park provisto de un programa para análisis electroquímicos: Echem.

- Bipotensiostato AFCBP1 acoplado a un rotor con velocidad modulada AFMSRCE ambos de Pine Instruments Co. 
Mundo Nano | CATÁLISIS EN MÉxICo | www.mundonano.unam.mx

10(18), enero-junio 2017 | DOI: 10.22201/ceiich.24485691e.2017.18.60111
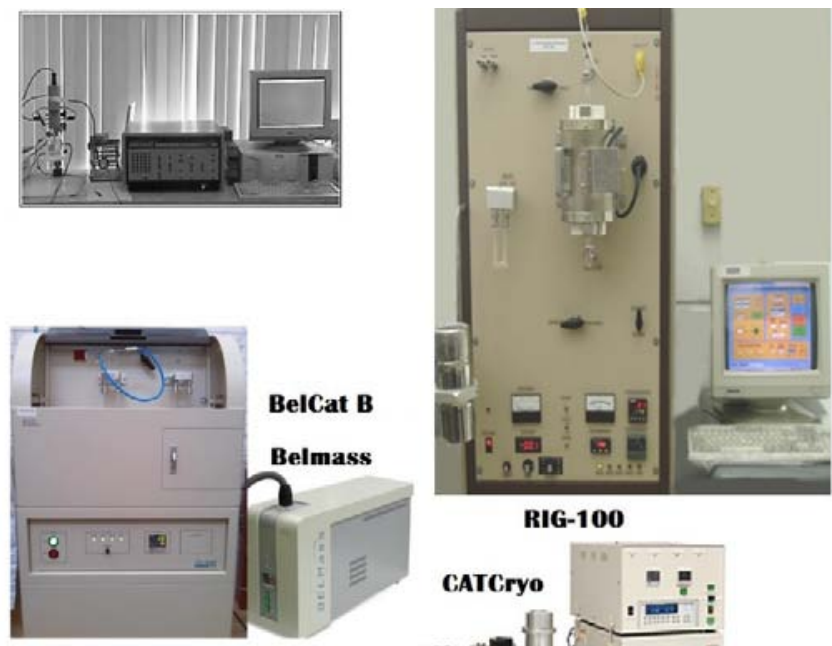

RIG-100

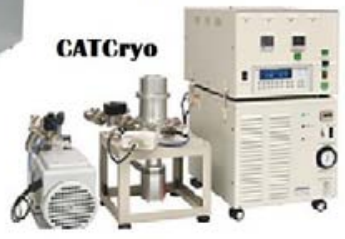

FIGURA 3. Equipos de caracterización de nanocatalizadores para la obtención de hidrógeno.

- Baño de agua con control de temperatura.

- Equipos para determinar área superficial por método BET; analizadores de poros, Bel Sorp max Bel Japan Inc.

- Equipo de infrarrojo,

- Analizadores termogravimétricos y calorímetros diferenciales de barrido

- Espectrómetro microRaman, LabRam 800 de Horiba con láser de Nd:YAG con emisión en $532 \mathrm{~nm}$.

- Espectrofluorómetro, Fluoromax de Horiba.

Adicionalmente, existen en el ININ laboratorios con equipos especializados destinados a la caracterización de nanomateriales: dos microscopios electrónicos de barrido (SEM), un microscopio electrónico de transmisión (TEM); cada uno tiene acoplado un espectrómetro de dispersión de energía de rayos-X para análisis químico elemental (EDS). También se cuenta con un sistema para análisis elemental por microfluorescencia de rayos-X ( $\mu$-EDXRF) y un sistema de retrodispersión de difracción de electrones (EBSD). Se tiene una cámara digital sCMOS para la adquisición de imágenes con resolución atómica, patrones de difracción de área selecta (SAED) y nanodifracción (NBD) en TEM. Un microscopio de sonda de barrido con capacidad para hacer microscopía de fuerza atómica y de tunelamiento. Se cuenta con un difractómetro de rayos-X para polvos con óptica de Bragg-Brentano y un difractómetro de rayos-X de óptica múltiple, que permite realizar distintas técnicas 


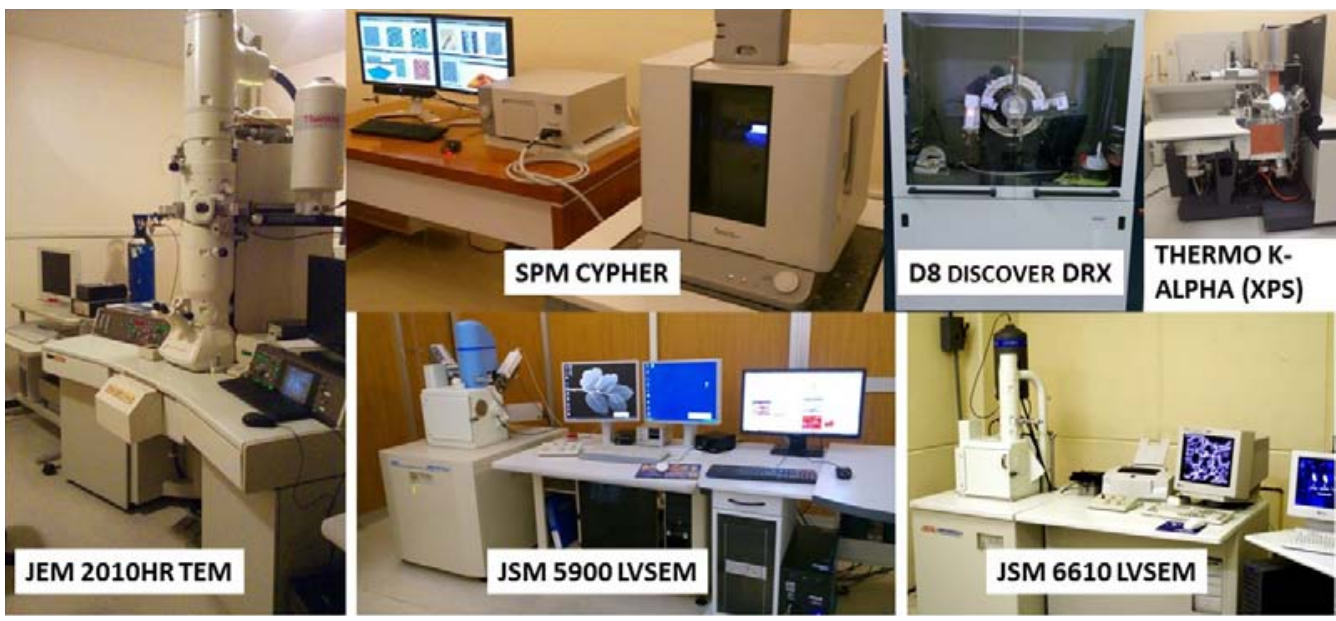

FIGURA 4. Equipos disponibles para la caracterización de los materiales catalíticos.

de análisis cristalográfico como difracción de amplio rango, microdifracción $(\mu-\mathrm{XRD})$, dispersión de rayos-X a bajo ángulo (SAXS), cuenta además con una platina de calentamiento. Se tienen equipos de preparación de muestras para microscopía electrónica: ultramicrotomo, electropulidor Twin-Jet, equipos para recubrir la superficie con carbono y oro de muestras no conductoras (Mundo Nano. Revista Interdisciplinaria en Nanociencias y Nanotecnología, vol. 9, núm.17, 2016: 159-174). Se cuenta también con un equipo para espectroscopía fotoelectrónica de rayos-X (XPS).

\section{Evaluación de propiedades catalíticas}

- Reactores de cuarzo de lecho fijo en flujo dinámico.

- Reactores de fotocatálisis.

- Celdas de combustible tipo PEM.

- Celdas de combustible alcalinas.

- Celdas de carbonatos fundidos.

\section{Docencia y formación de recursos humanos}

El ININ no otorga grados académicos y no cuenta con programas de posgrado, no obstante, como estrategia para formar recursos humanos, la principal colaboración con instituciones externas ha sido a través de la codirección de tesis con académicos adscritos a programas académicos en química, ambiental y ciencia de materiales con formación en temas de catálisis de las instituciones siguientes: 
- Universidad Nacional Autónoma de México.

- Universidad Autónoma del Estado de México.

- Benemérita Universidad Autónoma de Puebla.

- Universidad Autónoma Metropolitana (campus Lerma e Iztapalapa).

- Universidad Autónoma de Querétaro.

- Universidad Tecnológica del Valle de Toluca.

- Instituto Tecnológico de Toluca.

- Tecnológico de Estudios Superiores de San Felipe del Progreso.

- Tecnológico de Estudios Superiores de Jocotitlán.

- Tecnológico de Tuxtla Gutiérrez Chiapas.

- Centro de Investigación y Estudios Avanzados.

- Instituto Politécnico Nacional.

- Escuela Nacional Superior de Química de París (École Nationale Supérieure de Chimie de Paris ENSCP-Paris-Tech).

- Universidad de Columbia Británica, Vancouver, Canadá.

La contribución del ININ en la formación de recursos humanos en temas relacionados con la catálisis ha dado como resultado la formación de un buen número de estudiantes de licenciatura, maestría, doctorado y posdoctorado.

\section{Principales logros del ININ en catálisis}

El trabajo de investigación en catálisis realizado en el ININ se puede resumir en los siguientes logros:

- Desarrollo de catalizadores unidimensionales 1D (rods) con morfologías y planos cristalográficos preferenciales para la generación de hidrógeno a partir del reformado de alcoholes y metano.

- Se ha logrado obtener de forma sistemática películas de $\mathrm{N}_{-} \mathrm{TiO}_{2}$ tanto en la fase de anatasa como combinadas de anatasa/rutilo, las cuales fueron dopadas con nitrógeno y en pruebas preliminares, una aplicación de las mismas ha sido en la degradación del azul de metileno en concentraciones de $5 \mathrm{ppm}$ del $15 \%$ solo con $\mathrm{TiO}_{2}$ y se incrementó al $35 \%$ con $\mathrm{N}^{-\mathrm{TiO}_{2}}$.

- El uso de radiocatálisis ha demostrado ser efectivo en la degradación de colorantes (rojo Congo y azul de metileno), organoclorados (4-clorofenol) y contaminantes emergentes (diclofenaco), que depende de las condiciones de reacción. Se trataron con éxito muestras industriales de líquidos penetrantes y aguas residuales de una planta de tratamiento con alto contenido de demanda química de oxígeno (DQO).

Obtención de nanomateriales capaces de absorber luz visible para activarse como fotocatalizadores y emplearse en la degradación de compuestos orgánicos en solución, como fármacos o colorantes presentes en aguas residuales, utilizando luz solar. 

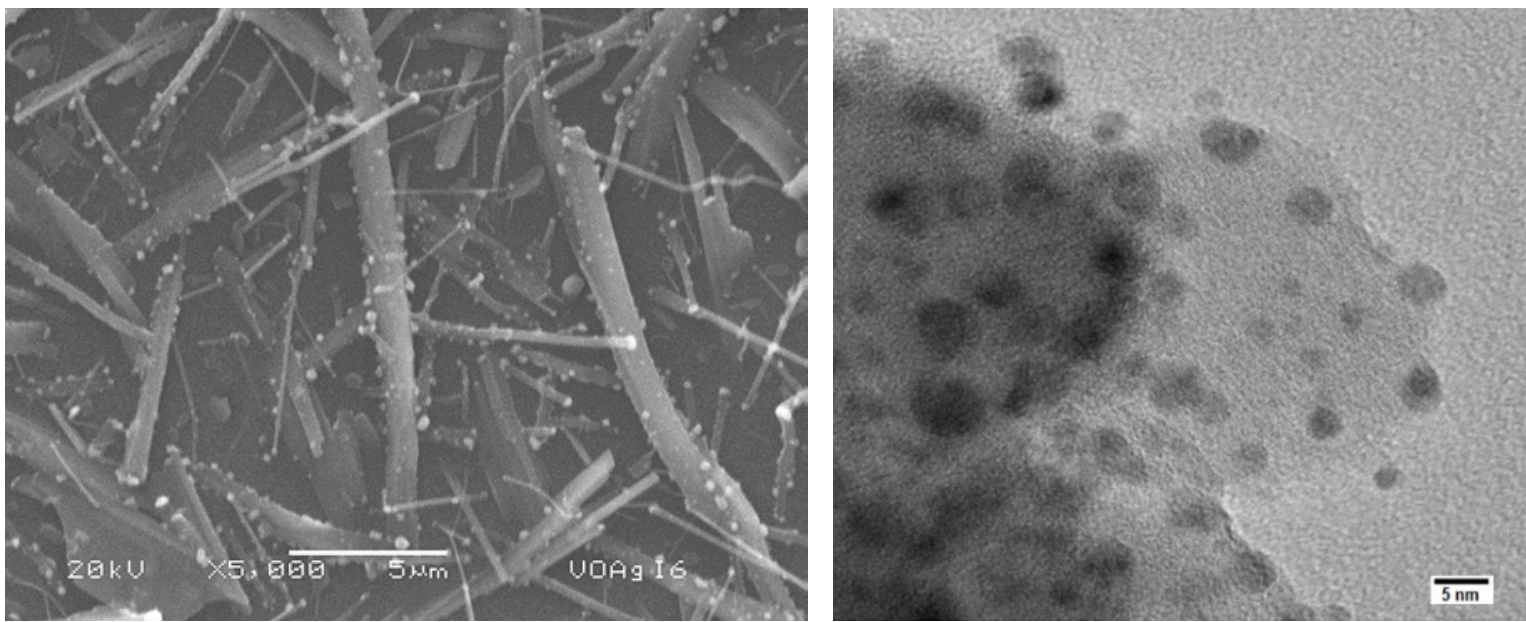

FIGURA 5. (Izq.) Nanobarras de vanadato de plata $\left(\mathrm{AgVO}_{3}\right)$ decoradas con nanopartículas de plata para aplicaciones en fotocatálisis. (Dcha.) Imagen de microscopía de trasmisión (TEM) de nanopartículas de plata sintetizadas por sol-gel para las reacciones de reducción y desprendimiento de oxígeno.

- Preparación y caracterización de nanopartículas de Au, Ag y Bi por ablación láser en vacío y en medio líquido, logrando obtener nanopartículas y nanoestructuras novedosas con un buen control en el tamaño y forma y por lo tanto en sus propiedades físicas.

- Preparación de nanoestructuras bidimensionales por ablación en medio líquido mediante un sistema asistido por ultrasonido. Algunas de estas nanoestructuras se han soportado sobre películas delgadas de $\mathrm{TiO}_{2}$ con el propósito de mejorar su desempeño fotocatalítico en la degradación de moléculas orgánicas.

- Preparación de películas delgadas bifuncionales para uso en celdas de combustible regenerativas y que pueden comportarse como celda de combustible al cambiar su polaridad, llevando a cabo la reacción de reducción de oxígeno de manera directa sin la presencia de intermediarios de reacción.

- Preparación de bicapas $\mathrm{TiO}_{2} / \mathrm{ITO} /$ vidrio por ablación láser sobre fibras ópticas de $500 \mu \mathrm{m}$ de diámetro para sistemas electrocatalíticos.

- Preparación de nanomateriales híbridos formados por nanopartículas de $\mathrm{Au}, \mathrm{Ag}, \mathrm{Bi}, \mathrm{Zn}$, soportadas o embebidas en películas delgadas de $\mathrm{TiO}_{2}$ y $\mathrm{V}_{2} \mathrm{O}_{5}$, o incluso en otros materiales

- Obtención de aleaciones o de metales nanopartículados por aleado mecánico, por sol-gel o por la técnica de microondas para ser utilizadas en celdas de combustible.

- Modificar la superficie del níquel con óxidos de metales de transición y/o lantánidos para incrementar el tiempo de vida del cátodo en las celdas de carbonatos fundidos. 


\section{Perspectivas sobre el estudio en catálisis}

Los grupos de investigación en catálisis del ININ consideran que la investigación que realizan es promisoria y tiene aplicaciones potenciales a nivel industrial, ya que pueden contribuir al desarrollo tecnológico que México requiere. En este sentido, se plantean algunas de estas perspectivas:

- Reducción de la contaminación ambiental mediante el desarrollo de nuevas fuentes integrales de energía limpia (hidrógeno) utilizando catalizadores "a la medida", esto es, nanomateriales con propiedades específicas para la aplicación particular. Lo anterior, con el propósito de promover la cultura de desarrollo sustentable en materia de energía y ambiente.

- Las reacciones inducidas sobre superficies sólidas por fotones y por radiación de alta energía son de particular interés para la catálisis y la ciencia de materiales. Sin embargo, en contraste con la gran cantidad de investigaciones sobre catálisis en fase gas-sólido y líquidosólido utilizando fotones (fotocatálisis), la química de radiaciones ionizantes en sistemas heterogéneos (radiocatálisis) está lejos de ser comprendida. Se plantea contribuir en el estudio de radiaciones ionizantes y catalizadores para modificar las reacciones de degradación de compuestos orgánicos y poder entender mejor los procesos por su mecanismo y cinética.

- Las películas formadas sobre los sustratos de vidrio y silicio se espera aplicarlas, entre otros, en un futuro cercano, en la remoción de contaminantes tóxicos en aire y agua, en hospitales y la industria de los alimentos debido a sus propiedades bactericidas, degradación del azul de metileno empleado en la industria textil, generación de hidrógeno por separación de agua. En un futuro cercano las películas de $\mathrm{N}-\mathrm{TiO}_{2}$ serán co-dopadas con metales, esto con el objetivo de reducir la energía del ancho de banda prohibida y lograr una mayor eficiencia en la catálisis.

- Modificar el $\mathrm{TiO}_{2}$ y $\mathrm{V}_{2} \mathrm{O}_{5}$ con dos o más metales con el propósito de mejorar su desempeño fotocatalítico en la degradación de fármacos.

- Explorar otras aplicaciones potenciales de los materiales que ya se preparan, en particular, en el área de fotocatálisis para la obtención de hidrógeno o para el tratamiento de aguas residuales, trabajando con sistemas más cercanos a los reales.

- Obtención de películas por la técnica de depósito atómico (atomic layer deposition) para entender y prevenir la degradación del cátodo de níquel en celdas de carbonatos fundidos.

- Iniciar el trabajo de desarrollo de patentes en los casos susceptibles de poder lograrse. 
A manera de conclusión, queremos señalar que la catálisis en México se realiza de forma aislada por diferentes grupos en universidades e institutos de investigación, sin embargo, es necesario impulsar mecanismos de interacción entre los diferentes grupos y de esta forma avanzar como un frente común que permita resolver problemas ambientales y energéticos en nuestro país, para desarrollar tecnologías nacionales que redunden en beneficios sociales y que permitan un mejor progreso de la nación. En particular, en el ININ, se ha conformado un grupo de trabajo interinstitucional que realiza investigación científica de alta calidad en la síntesis y caracterización de nanomateriales con aplicaciones potenciales en diversas áreas de la catálisis, propiciando el intercambio de experiencias entre los diferentes investigadores que participan en diferentes campos de investigación en catálisis. Los resultados del trabajo de investigación realizado en el ININ se reflejan en la generación de nuevos conocimientos en las áreas de catalizadores y electrocatalizadores para emplearlos en la generación de combustibles alternos al petróleo, así como en la síntesis de nuevos materiales fotocatalíticos para tratamiento de agua; los cuales son presentados en foros científicos llevados a cabo por diferentes organizaciones científicas nacionales, entre ellas, la Academia de Catálisis A.C. (ACAT) y la Sociedad Mexicana del Hidrógeno $(\mathrm{SMH})$. Adicionalmente, redundan en la formación de recursos humanos altamente calificados en temas relacionados con la catálisis.

\section{Referencias recientes (o más relevantes) del ININ en investigación en catálisis}

R. Pérez-Hernández, A. Gutiérrez-Martínez, M. E. Espinosa-Pesqueira, Ma. L. Estanislao, J. Palacios. Effect of the bimetallic Ni/Cu loading on the $\mathrm{ZrO}_{2}$ support for $\mathrm{H}_{2}$ production in the autothermal steam reforming of methanol. Catalysis Today, 250, (2015), 166-172.

R. Pérez-Hernández, G. Mondragón-Galicia, A. Allende Maravilla y J. Palacios. Nanodimensional $\mathrm{CeO}_{2}$ nanorods for high Ni loading catalysts: $\mathrm{H}_{2}$ production by autothermal steam reforming of methanol reaction. Physical Chemistry Chemical Physics, 15 (30) (2013), 12702-12708.

E. Casillas, F. Tzompantzi, S. G. Castellanos, G. Mendoza-Damián, R. Pérez-Hernández, A. López-Gaona, A. Barrera. Promotion effect of $\mathrm{ZnO}$ on the photocatalytic activity of coupled $\mathrm{Al}_{2} \mathrm{O}_{3}-\mathrm{Nd}_{2} \mathrm{O}_{3}-\mathrm{ZnO}$ composites prepared by the sol - gel method in the degradation of phenol, Journal Applied Catalysis B: Environmental, 208, (2017), 161-170.

Gilberto Mondragón-Galicia, Claudia Gutiérrez-Wing, María Eufemia Fernández García, Demetrio Mendoza-Anaya y Raúl Pérez-Hernández. Ag nanowires as precursors to synthesize Ag-ZnO nanostructured brushes, RSC Advances, 5, (2015), 42568-12571.

Raúl Pérez-Hernández, Demetrio Mendoza-Anaya, Albina Gutiérrez Martínez y Antonio Gómez-Cortés. Capítulo 6: Catalytic steam reforming of methanol 
to produce hydrogen on supported metal catalysts. pp. 149-256. http:// dx.doi.org/10.5772/49965. En Hydrogen Energy-Challenges and Perspectives. http://dx.doi.org/10.5772/2824. Edited by Dragica Minić. Published by InTech-2012.

A. Rodríguez-Méndez, I. Viveros-Palma, C. Guzmán, Eduardo A. Elizalde-Peña, L. Escobar-Alarcón, Marina Vega, Josefina Alvarado Rivera, K. Esquivel. Effluent disinfection of real wastewater by $\mathrm{Ag}-\mathrm{TiO}_{2}$ nanoparticles photocatalysis, Journal of Nanoscience and Nanotechnology, 17 (1), 711-719, (2017).

Emmanuel Velarde Granados, Luis Escobar Alarcón, Dora Alicia Solís Casados, Cecilia Encarnación Gómez, Oscar Fernando Olea Mejía, Manuel Espinosa Pesqueira, Emmanuel Haro Poniatowski. Nanoestructuras basadas en Bi soportadas en películas delgadas de $\mathrm{TiO}_{2}$ y su desempeño fotocatalítico, Superficies y Vacío, 28 (2) 54-60, (2015).

Jonatán Pérez-Alvarez, Dora A. Solís-Casados, Saúl Romero, Luis Escobar-Alarcón. Photocatalytic degradation of malachite green dye and pharmaceuticals using Co: $\mathrm{TiO}_{2}$ thin films, Advanced Materials Research, 976, 212-216, (2014).

F. Gonzalez-Zavala, L. Escobar-Alarcón, D. A. Solís-Casados, C. Rivera-Rodríguez, R. Basurto, E. Haro-Poniatowski. Preparation of vanadium oxide thin films modified with Ag using a hybrid deposition configuration, Applied Physics A, 122: 461, (2016).

Escobar-Alarcón L., Morales-Ramírez C., Klimova T., Escobar-Aguilar J., Romero S., Solís-Casados D. Preparation and characterization of $\mathrm{Al}_{2} \mathrm{O}_{3}-\mathrm{MgO}$ catalytic supports modified with lithium, Fuel, 110, 278-285, (2013).

J. Jiménez-Becerril, A. Martínez-Hernández, F. Granados-Correa, R. E. Zavala-Arce. Methylene blue and 4-chlorophenol photodegradation using gamma-irradiated titanium oxide, J. Chem. Soc. Pak., 2013, 35 (1), 23-26

J. C. González-Juárez, J. Jiménez-Becerril, J. Cejudo-Álvarez. Degradation of 4-chlorophenol by gamma radiation of 137 Cs and X-rays, J. Mex. Chem. Soc., (2010), 54 (1), 2-6.

J. C. González-Juárez, J. Jiménez-Becerril. Gamma radiation-induced catalytic degradation of 4-chlorophenol using $\mathrm{SiO}_{2}, \mathrm{TiO}_{2}$, and $\mathrm{Al}_{2} \mathrm{O}_{3}$. Rad. Phys. Chem., (2006), 75, 768-772.

J. Jiménez-Becerril, A. Moreno-López, M. Jiménez-Reyes. Radiocatalytic degradation of dissolved organic compounds in wastewater. NUKLEONIKA, (2016), 61 (4), 473-476.

J. Jiménez-Becerril, J. C. González-Juárez, R. Contreras-Bustos. Penetrant liquid waste degradation by radiocatalysis, J. Residuals Sci. Tech., (2013), 10 (4), 171-177.

R. Valencia-Alvarado, A. de la Piedad-Beneitez, R. López-Callejas, A. Mercado-Cabrera, R. Peña-Eguiluz, A. E. Muñoz-Castro, B. G. Rodríguez-Méndez, y J. M. de la Rosa-Vázquez. $\mathrm{TiO}_{2}$ thin and tick films grown on $\mathrm{Si} /$ glass by sputtering of Titanium targets in an RF inductively coupled plasma, Journal of Physics: Conference Series, 591, (2015), 012042.

R. Valencia-Alvarado, A. de la Piedad-Beneitez, R. López-Callejas, B. G., RodríguezMéndez, A. Mercado-Cabrera, R. Peña-Eguiluz, A. E. Muñoz-Castro y J. M. de 
la Rosa-Vázquez. Sequential processes to produce $\mathrm{N}-\mathrm{TiO}_{2}$ films through $\mathrm{Rf}$ plasmas, MATEC Web of Conferences, 67, (2016), 06075.

R. Valencia-Alvarado, A. de la Piedad-Beneitez, R. López-Callejas, B. G., RodríguezMéndez, A. Mercado-Cabrera, R. Peña-Eguiluz, A. E. Muñoz-Castro, y J. M. de la Rosa-Vazquez. Titanium dioxide films grown on glass and silicon by sputtering, Kasmera Journal, 44-1, (2016), 423-441.

\section{Sitios de interés relacionados de ININ}

- Instituto Nacional de Investigaciones Nucleares | Gobierno | gob.mx, Website title: Gob.mx, URL:

<https://www.gob.mx/inin/>

- Facebook:

<https://www.facebook.com/ININmx-457060114342632/>

- Twitter:

<https://twitter.com/inin_mx>

- Instagram:

<https://www.instagram.com/ininmx/>

- YouTube:

<https://www.youtube.com/channel/UCd305j-

hk0Pnb2QK0Od9ryQ> 